\title{
An Analytical Solution to the Heat Transfer Problem in Shercliff Flow
}

\author{
Michael J Bluck, Michael J Wolfendale, Andrew J Marquis \\ Mechanical Engineering Dept. Imperial College, London
}

\begin{abstract}
The study of flow in a rectangular duct, subject to a strong transverse magnetic field is of interest in a number of applications. An important application of such flows is in the context of coolants, where the principle issue of interest is convective heat transfer. For fully developed laminar flows, the problem can be characterised in terms of two coupled partial differential equations. In the case of perfectly electrically insulating boundaries, there is a well known analytical solution due to Shercliff, which provides the velocity and induced magnetic field profiles. In this paper, we demonstrate analytical solutions to $H_{1}$ and $H_{2}$ heat transfer problems for the Shercliff case in rectangular ducts and obtain temperature profiles and corresponding Nusselt numbers as functions of aspect ratio and Hartmann number.
\end{abstract}

Keywords:

Magnetohydrodynamics, Nusselt number, Heat transfer, Shercliff flow

\section{Introduction}

The flow in a rectangular duct, subject to strong transverse magnetic fields, is of significant interest in fusion applications due to the use of liquid metal coolants employed in some fusion blanket designs. Depending on the circumstances, this magnetohydrodynamic problem may be simplified by assuming a laminar fully-developed flow with perfectly electrically insulating walls. The problem then reduces to two coupled partial differential equations, whose solution was first obtained by Shercliff [1]. Shercliff obtained explicit analytical solutions for the velocity and magnetic field profiles for this case and his work was subsequently extended to the case of imperfectly and perfectly conducting walls by Hunt $[2,3]$.

In the context of fusion blankets, of equal or greater importance is the concomitant heat transfer, as the extraction of heat is one of the main roles of the blanket itself. Despite the existence of analytical solutions for the velocity profile, there is as yet (to the authors knowledge) no corresponding solution to the heat transfer problem for the Shercliff case (and indeed the Hunt cases). Such solutions exist for flow between parallel plates and flows in circular channels [4, 5], and for 1-D heat transfer [6]. There are also some experimental and many numerical studies of heat transfer for Shercliff and related cases $[7,8,9,10,11,12]$. It should be noted that even though numerical solutions exist, analytical solutions play an important role in the validation of such computational codes and can give significant insight into the underlying physics, as well as providing approximate parameters for 1-D thermal-hydraulic systems codes. In this article we extend an analytical solution of the temperature profile in rectangular ducts for both the $\mathrm{H} 1$ and $\mathrm{H} 2$ heat transfer cases, already well developed for the non-MHD case, to the electrically insulating wall MHD case (Shercliff flow). To the author's knowledge this is novel.

\section{Problem Formulation}

Referring to Fig. 1, the momentum equation in a fully developed MHD flow in a rectangular duct of size $-a d_{h} \leq X \leq a d_{h}$ and $-b d_{h} \leq Y \leq b d_{h}$ (where $d_{h}$ is the hydraulic diameter), subject to an applied $X$-directed magnetic field $B_{x}^{0}$ is given by

$$
v\left(\frac{\partial^{2} U}{\partial X^{2}}+\frac{\partial^{2} U}{\partial Y^{2}}\right)-\frac{1}{\rho} \frac{\partial p}{\partial Z}+\frac{1}{\rho \mu} \frac{\partial B_{z}}{\partial X} B_{x}^{0}=0
$$

The flow of conducting fluid generates an induced magnetic field $B_{z}$, satisfying

$$
\frac{1}{\mu \sigma}\left(\frac{\partial^{2} B_{z}}{\partial X^{2}}+\frac{\partial^{2} B_{z}}{\partial Y^{2}}\right)+B_{x}^{0} \frac{\partial U}{\partial X}=0
$$

Where $U$ is the velocity, $v$ is the kinematic viscosity, $\mu$ the magnetic permeability, $\rho$ the density and $\sigma$ the electrical conductiv-

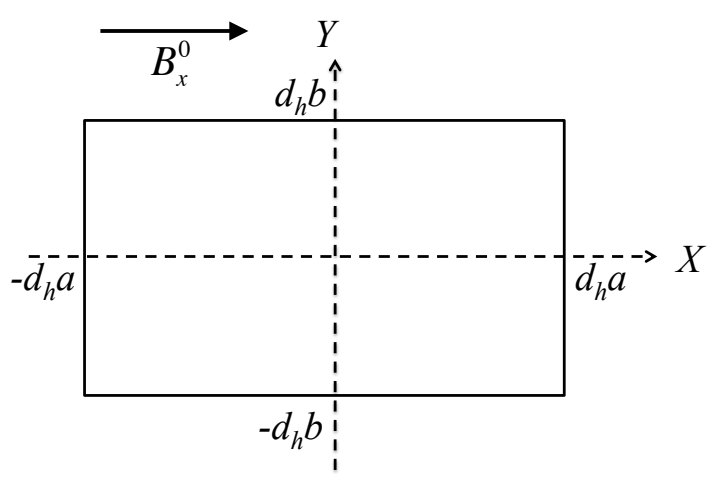

Figure 1: Duct coordinate system 


\section{Nomenclature}

$\Gamma \quad$ Wetted perimeter (m).

$\mu \quad$ Fluid magnetic permeability $\left(\mathrm{Hm}^{-1}\right)$.

$v \quad$ Kinematic viscosity $\left(\mathrm{m}^{2} \mathrm{~s}^{-1}\right)$.

$\rho \quad$ Fluid density $\left(\mathrm{kgm}^{-3}\right)$.

$\sigma \quad$ Fluid electrical conductivity $\left(\mathrm{Sm}^{-1}\right)$.

A Duct cross-sectional area $\left(\mathrm{m}^{2}\right)$.

$a \quad$ Duct half-width (m).

$b \quad$ Duct half-height (m).

$B_{x}^{0} \quad$ Applied $x$-directed magnetic field $\left(\mathrm{Wb} \mathrm{m}^{-2}\right)$.

$B_{z} \quad$ Induced magnetic field $\left(\mathrm{Wb} \mathrm{m}^{-2}\right)$.

$d_{h} \quad$ Hydraulic diameter (m).

$h \quad$ Dimensionless magnetic field.

$\mathrm{Ha}$ Hartmann number.

$\begin{array}{ll}\mathrm{Hg} & \text { Hagen number. } \\ \mathrm{Nu} & \text { Nusselt number. } \\ p & \text { Pressure }(\mathrm{Pa}) . \\ \mathrm{Pr} & \text { Prandtl number. } \\ q^{\prime \prime} & \text { Heat flux }\left(\mathrm{Wm}^{-2}\right) . \\ R e & \text { Reynolds number. } \\ T & \text { Temperature profile }(\mathrm{K}) . \\ t & \text { Dimensionless temperature. } \\ T_{m} & \text { Bulk temperature }(\mathrm{K}) . \\ T_{w} & \text { Wall temperature }(\mathrm{K}) . \\ U & \text { Velocity profile }\left(\mathrm{ms}^{-1}\right) . \\ u & \text { Dimensionless velocity. } \\ U_{m} & \text { Mean velocity }\left(\mathrm{ms}^{-1}\right) .\end{array}$

$\mathrm{Hg} \quad$ Hagen number.

$\mathrm{Nu} \quad$ Nusselt number.

$q^{\prime \prime} \quad$ Heat flux $\left(\mathrm{Wm}^{-2}\right)$.

Re Reynolds number.

$T \quad$ Temperature profile (K).

Dimensionless temperature.

ure $(\mathrm{K})$.

Wall temperature $(\mathrm{K})$.

$U_{m} \quad$ Mean velocity $\left(\mathrm{ms}^{-1}\right)$
Non-dimensionalising, by setting

$$
\begin{aligned}
& x=\frac{X}{d_{h}}, y=\frac{Y}{d_{h}}, z=\frac{Z}{d_{h}} \\
& u=\frac{U}{U_{m}}
\end{aligned}
$$

where

$$
U_{m}=\frac{1}{A} \int_{A} U d A
$$

and

$$
h=\frac{1}{\mu} \frac{1}{\sqrt{\rho v \sigma}} \frac{1}{U_{m}} B_{z}
$$

we obtain

$$
\frac{\partial^{2} u}{\partial x^{2}}+\frac{\partial^{2} u}{\partial y^{2}}+H a \frac{\partial h}{\partial x}=\frac{H g}{R e}
$$

where

$$
H a=B_{x}^{0} d_{h} \sqrt{\frac{\sigma}{\rho v}}
$$

and the Hagen number is defined as

$$
H g=\frac{(\partial p / \partial Z) d_{h}^{3}}{\rho v^{2}}
$$

The no-slip condition requires that $u=0$ at the wall. The induced magnetic field $h$ satisfies

$$
\frac{\partial^{2} h}{\partial x^{2}}+\frac{\partial^{2} h}{\partial y^{2}}+H a \frac{\partial u}{\partial x}=0
$$

in the fluid region. For the Shercliff problem considered here, the induced magnetic field vanishes at the wall. The solution to this problem is well known, and is given in the appendix for the case.
In the following we consider the energy equation, which in steady state, fully developed flow, can be written as

$$
\frac{v}{P r}\left(\frac{\partial^{2} T}{\partial X^{2}}+\frac{\partial^{2} T}{\partial Y^{2}}\right)=U \frac{\partial T}{\partial Z}
$$

For now, we leave this equation in its dimensional form. The process of non-dimensionalization differs markedly between $H_{1}$ and $H_{2}$ cases and is dealt with at the beginning of sections 3.1 and 3.2 for the $H_{1}$ and $H_{2}$ cases, respectively.

\section{Analytical Solution}

\section{1. $H_{1}$ Heat transfer case}

The $H_{1}$ transfer case describes circumstances where the heat flux is uniform in the axial direction and the wall temperature $T_{w}$ is uniform in the peripheral direction. Under the conditions of fully developed Shercliff flow, it can be assumed that

$$
\frac{\partial T}{\partial Z}=\frac{d T_{m}}{d Z}=\text { const }
$$

where the bulk temperature $T_{m}$ is defined as

$$
T_{m}=\frac{\int_{A} U T d A}{\int_{A} U d A}
$$

We non-dimensionalize as before, with the non-dimensional temperature profile $t(x, y)$ being defined by

$$
t=\frac{T}{\left(d T_{w} / d Z\right) d_{h}}
$$

Inserting these into equation (11) gives

$$
\frac{\partial^{2} t}{\partial x^{2}}+\frac{\partial^{2} t}{\partial y^{2}}=(\operatorname{RePr}) u
$$


We now proceed to determine the non-dimensional temperature profile $t(x, y)$ by decomposing the solution into a particular integral and a general solution. We obtain the following particular integral, which satisfies (15).

$$
t_{p}(x, y)=\operatorname{Hg} \operatorname{Pr} \sum_{n=1}^{\infty} f_{n}(x) \cos \lambda_{n} y
$$

where

$$
\begin{array}{r}
f_{n}(x)=\frac{k_{n}}{\lambda_{n}^{2} b}\left(-\frac{1}{\lambda_{n}^{2}}-\frac{\sinh p_{n 2} a \cosh p_{n 1} x}{\left(p_{n 1}^{2}-\lambda_{n}^{2}\right) \sinh \left(p_{n 2}-p_{n 1}\right) a}\right. \\
\left.+\frac{\sinh p_{n 1} a \cosh p_{n 2} x}{\left(p_{n 2}^{2}-\lambda_{n}^{2}\right) \sinh \left(p_{n 2}-p_{n 1}\right) a}\right)
\end{array}
$$

where $p_{n 1}, p_{n 2}$ and $\lambda_{n}$ are defined in the appendix. This expression vanishes on the upper and lower walls $(y= \pm b)$, but does not vanish at $x= \pm a$.

It can be easily shown that there is a general solution to the Laplace equation on the same geometry, which vanishes on $y=$ $\pm b$, but is non-zero on $x= \pm a$ :

$$
\begin{aligned}
t_{l}(x, y) & =\operatorname{Hg} \operatorname{Pr} \sum_{n=1}^{\infty}\left(a_{n} \sinh \lambda_{n}(x-a)\right. \\
& \left.+b_{n} \sinh \lambda_{n}(x+a)\right) \cos \lambda_{n} y
\end{aligned}
$$

for constants $a_{n}$ and $b_{n}$. We will now require that $t_{p}+t_{l}$ vanishes on $x= \pm a$. Using these expressions we can calculate $a_{n}$ and $b_{n}$ as

$$
\begin{aligned}
& a_{n}=\frac{f_{n}(-a)}{\sinh 2 \lambda_{n} a} \\
& b_{n}=-\frac{f_{n}(a)}{\sinh 2 \lambda_{n} a}
\end{aligned}
$$

Note also that $f_{n}(-a)=f_{n}(a)$, so $a_{n}=-b_{n}$.

Given these results, we can re-dimensionalize and obtain the following solution to equation (11) satisfying $T=T_{w}$ at the boundary:

$$
T(x, y)=T_{w}+d_{h} H g \operatorname{Pr} \frac{d T_{m}}{d Z} \sum_{n=1}^{\infty} g_{n}(x) \cos \lambda_{n} y
$$

where

$$
g_{n}(x)=f_{n}(x)+\frac{f_{n}(a)}{\sinh 2 \lambda_{n} a}\left(\sinh \lambda_{n} \pi(x-a)-\sinh \lambda_{n}(x+a)\right)(21)
$$

from which the local Nusselt number $N u_{\mathbf{n}}$ for a wall with unit normal $\mathbf{n}$ as

$$
N u_{\mathbf{n}}=\frac{d_{h} \mathbf{n} \cdot \nabla T}{\left(T_{w}-T_{m}\right)}
$$

and the overall mean Nusselt number as

$$
\overline{N u}=\frac{d_{h}}{\Gamma} \frac{\int_{\partial A} \mathbf{n} \cdot \nabla T d s}{\left(T_{w}-T_{m}\right)}
$$

where $\Gamma$ is the wetted perimeter.

\section{2. $\mathrm{H}_{2}$ Heat transfer case}

The $\mathrm{H}_{2}$ transfer case describes circumstances where the heat flux is uniform in the axial direction and is also uniform in the peripheral direction. We follow an analysis similar to [13]. The same magnetohydrodynamic conditions arise as in the $H_{1}$ case, but the treatment of the energy equation differs somewhat. Due to the uniform peripheral and axial heat flux $q^{\prime \prime}$ we can perform an energy balance:

$$
q^{\prime \prime} \Gamma d Z=\rho c_{p} A U_{m} d T
$$

from which it follows that

$$
\frac{\partial T}{\partial Z}=\frac{d T_{m}}{d Z}=\frac{q^{\prime \prime} \Gamma}{\rho c_{p} A U_{m}}=\frac{4 q^{\prime \prime}}{\rho c_{p} d_{h} U_{m}}
$$

In this case we define the non-dimensional temperature profile $t(x, y)$ as

$$
t=\frac{k}{q^{\prime \prime} d_{h}}\left(T-T_{m}\right)
$$

Inserting these into equation (11) gives

$$
\frac{\partial^{2} t}{\partial x^{2}}+\frac{\partial^{2} t}{\partial y^{2}}=4 u
$$

The boundary conditions at the wall are

$$
k \frac{\partial T}{\partial n}=q^{\prime \prime}
$$

which take the non-dimensional form

$$
\frac{\partial t}{\partial n}=1
$$

Following [13] we homogenise this equation by defining $\theta(x, y)$ as

$$
\theta(x, y)=t(x, y)-\left(\frac{x^{2}}{2 a}+\frac{y^{2}}{2 b}\right)
$$

Substituting for $t$ into equation (27) and considering only one quarter of the duct due to symmetry we obtain

$$
\begin{gathered}
\frac{\partial^{2} \theta}{\partial x^{2}}+\frac{\partial^{2} \theta}{\partial y^{2}}=4(u-1) \\
\frac{\partial \theta}{\partial x}=0, \text { on } x=0 \\
\frac{\partial \theta}{\partial x}=0, \text { on } x=a \\
\frac{\partial \theta}{\partial y}=0, \text { on } y=0 \\
\frac{\partial \theta}{\partial y}=0, \text { on } y=b
\end{gathered}
$$

To solve this equation, we begin by considering the homogeneous form and decomposing via a separation of variables approach. As shown in [13], the solution to (31) is of the form

$$
\theta(x, y)=X_{0}(x)+\sum_{n=1}^{\infty} X_{n}(x) \cos \left(\frac{n \pi y}{b}\right)
$$


Substituting this expression into equation (31) gives

$$
\begin{array}{r}
X_{0}^{\prime \prime}(x)+\sum_{n=1}^{\infty}\left(X_{n}^{\prime \prime}(x)-\left(\frac{n \pi}{b}\right)^{2} X_{n}(x)\right) \cos \left(\frac{n \pi y}{b}\right) \\
=4(u(x, y)-1)
\end{array}
$$

Considered as a Fourier series, the zeroth order term is the mean value of the RHS of (37), so

$$
X_{0}^{\prime \prime}(x)=\frac{4}{b} \int_{0}^{b}(u(x, y)-1) d y
$$

which can be evaluated to give

$$
X_{0}^{\prime \prime}(x)=-4\left(1+\frac{1}{b} \frac{H g}{\operatorname{Re}} \sum_{n=1}^{\infty} \frac{(-1)^{n}}{\lambda_{n}} u_{n}(x)\right)
$$

Following two successive integrations with respect to $x$, we obtain

$$
\begin{aligned}
X_{0}(x) & =-2 x^{2}+c_{1} x+c_{2} \\
& +\frac{8}{b^{2}} \frac{H g}{\operatorname{Re}} \sum_{n=1}^{\infty} \frac{1}{\lambda_{n}^{4}}\left(\frac{x^{2}}{2}-\frac{\sinh p_{n_{2}} a \cosh p_{n_{1}} x}{p_{n_{1}}^{2} \sinh \left(p_{n_{2}}-p_{n_{1}}\right) a}\right. \\
& \left.+\frac{\sinh p_{n_{1}} a \cosh p_{n_{2}} x}{p_{n_{2}}^{2} \sinh \left(p_{n_{2}}-p_{n_{1}}\right) a}\right)
\end{aligned}
$$

It now remains to compute the $X_{n}$ terms, which is achieved by applying an orthogonality relation to equation (37) which gives

$$
X_{m}^{\prime \prime}(x)-\left(\frac{m \pi}{b}\right)^{2} X_{m}(x)=\frac{8}{b} \int_{0}^{b}(u(x, y)-1) \cos \left(\frac{m \pi y}{b}\right) d y
$$

which can be written as

$$
X_{m}^{\prime \prime}(x)-\left(\frac{m \pi}{b}\right)^{2} X_{m}(x)=\frac{8}{b} \frac{H g}{\operatorname{Re}} \sum_{n=1}^{\infty} \gamma_{m n} u_{n}(x)
$$

where

$$
\gamma_{m n}=\frac{1}{2}(-1)^{m+n-1}\left(\frac{1}{\lambda_{n}-\frac{m \pi}{b}}+\frac{1}{\lambda_{n}+\frac{m \pi}{b}}\right)
$$

The solution to this equation is decomposed into the sum of a particular integral $X_{m}^{p}$ and a general solution, $X_{m}^{g}$, to the homogeneous form of (42). The particular solution is given by

$$
X_{m}^{p}(x)=\sum_{n=1}^{\infty}\left(a_{m n}+b_{m n} \cosh p_{n_{1}} x+c_{m n} \cosh p_{n_{2}} x\right)
$$

Substituting this into (42) and equating coefficients gives

$$
\begin{aligned}
& a_{m n}=-\frac{8}{m^{2} \pi^{2}} \frac{H g}{\operatorname{Re}} \frac{k_{n}}{\lambda_{n}^{2}} \gamma_{m n} \\
& b_{m n}=-\frac{8}{b^{2}} \frac{H g}{\operatorname{Re}} \frac{k_{n}}{\lambda_{n}^{2}} \frac{\sinh p_{n_{2}} a}{\left(p_{n_{1}}^{2}-\left(\frac{m \pi}{b}\right)^{2}\right) \sinh \left(p_{n_{2}}-p_{n_{1}}\right) a} \gamma_{m n} \\
& c_{m n}=\frac{8}{b^{2}} \frac{H g}{\operatorname{Re}} \frac{k_{n}}{\lambda_{n}^{2}} \frac{\sinh p_{n_{1}} a}{\left(p_{n_{2}}^{2}-\left(\frac{m \pi}{b}\right)^{2}\right) \sinh \left(p_{n_{2}}-p_{n_{1}}\right) a} \gamma_{m n}
\end{aligned}
$$

The homogeneous solution of equation (42) is given by

$$
X_{m}^{g}(x)=d_{m} \sinh \frac{m \pi x}{b}+e_{m} \cosh \frac{m \pi x}{b}
$$

Substituting equation (48), (44) and (40) into (36), we obtain $\theta(x, y)$. Finally we apply the remaining boundary conditions, giving at $x=0$,

$$
c_{1}+\sum_{n=1}^{\infty} d_{n}\left(\frac{n \pi}{b}\right) \cos \left(\frac{n \pi y}{b}\right)=0 \Rightarrow c_{1}=d_{n}=0
$$

and at $x=a$, we have

$$
\begin{array}{r}
\sum_{n=1}^{\infty}\left(e_{n}\left(\frac{n \pi}{b}\right) \sinh \left(\frac{n \pi a}{b}\right)+\right. \\
\left.\sum_{m=1}^{\infty} b_{n m} p_{m_{1}} \sinh p_{m_{1}} a+c_{n m} p_{m_{2}} \sinh p_{m_{2}} a\right) \cos \left(\frac{n \pi y}{b}\right)=0
\end{array}
$$

So

$$
e_{n}=-\frac{b}{n \pi} \frac{\sum_{m=1}^{\infty} b_{n m} p_{m_{1}} \sinh p_{m_{1}} a+c_{n m} p_{m_{2}} \sinh p_{m_{2}} a}{\sinh \left(\frac{n \pi a}{b}\right)}
$$

Combining these results we obtain the following expression for $t$ in terms of the coefficients:

$$
\begin{aligned}
t(x, y) & =c_{2}+\left(\frac{x^{2}}{2 a}+\frac{y^{2}}{2 b}\right)-2 x^{2} \\
& +\frac{8}{b^{2}} \frac{H g}{R e} \sum_{n=1}^{\infty} \frac{1}{\lambda_{n}^{4}}\left(\frac{x^{2}}{2}-\frac{\sinh p_{n_{2}} a \cosh p_{n_{1}} x}{p_{n_{1}}^{2} \sinh \left(p_{n_{2}}-p_{n_{1}}\right) a}\right. \\
& \left.+\frac{\sinh p_{n_{1}} a \cosh p_{n_{2}} x}{p_{n_{2}}^{2} \sinh \left(p_{n_{2}}-p_{n_{1}}\right) a}\right) \\
& +\sum_{n=1}^{\infty}\left[e_{n} \cosh \frac{n \pi x}{b}\right. \\
& +\sum_{m=1}^{\infty}\left(a_{n m}+b_{n m} \cosh p_{m_{1}} x\right. \\
& \left.\left.+c_{n m} \cosh p_{m_{2}} x\right)\right] \cos \left(\frac{n \pi y}{b}\right)
\end{aligned}
$$

It can be shown that as the Hartmann number tends to zero, this expression tends to that given in [13].

It remains to compute the constant $c_{2}$. The determination of $c_{2}$ is achieved by applying the following constraint proposed in [13]:

$$
\int_{A} u t d A=0
$$

In that paper the authors justify this by demonstrating its application to a circular geometry. In fact, this can be very easily proven as follows: From (26) we see that

$$
u t=\frac{k}{q^{\prime \prime} d_{h}} \frac{U}{U_{m}}\left(T-T_{m}\right)=\frac{k}{q^{\prime \prime} d_{h} U_{m}}\left(U T-U T_{m}\right)
$$

Integrating over the cross-sectional area of the duct gives

$$
\int_{A} u t d A=\frac{k}{q^{\prime \prime} d_{h} U_{m}}\left(\int_{A} U T d A-T_{m} \int_{A} U d A\right)
$$


From (5) and (13) we see that

$$
\int_{A} u t d A=\frac{k}{q^{\prime \prime} d_{h} U_{m}}\left(A U_{m} T_{m}-T_{m} A U_{m}\right)=0
$$

and the constraint is proved. Once applied, this constraint determines $c_{2}$ and hence the non-dimensional temperature field $t$.

It is worth noting that the dimensional temperature $T$ is determined only up to an additive constant, eg. $T_{0}$, in the $H_{2}$ case. However, such an additive constant is not related to the constant $c_{2}$ in equation (52). This is clear, firstly, from the fact that if we have a temperature distribution $T^{\prime}=T+T_{0}$ where $T_{0}$ is a constant, then

$$
T_{m}^{\prime}=\frac{\int_{A} U\left(T+T_{0}\right) d A}{\int_{A} U d A}=T_{m}+T_{0}
$$

Equation (26), defining the non-dimensional temperature $t$ is then left unchanged since

$$
t^{\prime}=\frac{k}{q^{\prime \prime} d_{h}}\left(T^{\prime}-T_{m}^{\prime}\right)=\frac{k}{q^{\prime \prime} d_{h}}\left(T+T_{0}-T_{m}-T_{0}\right)=t
$$

We can conclude that adding a constant to the dimensional temperature $T$, does not affect $t$. Conversely, arbitrarily changing $c_{2}$ clearly changes $t$. Secondly, as we shall now show, we are not free to disregard the constant $c_{2}$, indeed, it is closely related to a non-dimensional bulk temperature $\tau_{m}$. Let us write (52) as $t=c 2+\tau$, then from the constraint (53),

$$
0=\frac{\int_{A} u t d A}{\int_{A} u d A}=\frac{\int_{A} u(c 2+\tau) d A}{\int_{A} u d A}=c_{2}+\frac{\int_{A} u \tau d A}{\int_{A} u d A}=c_{2}+\tau_{m}
$$

hence

$$
c_{2}=-\frac{\int_{A} u \tau d A}{\int_{A} u d A}=-\tau_{m}
$$

Thus, we are not free to choose $c_{2}$; it is determined from the velocity field $u$ and the solution (52). Of course, once $c_{2}$ has been obtained, we can determine the dimensional temperature field $T$ up to an additive constant, from equations (26) and (52).

The local Nusselt number at the wall can be determined from

$$
N u_{\mathbf{n}}=\frac{d_{h} \mathbf{n} \cdot \nabla T}{\left(T_{w}-T_{m}\right)}=\frac{d_{h} q^{\prime \prime}}{k\left(T_{w}-T_{m}\right)}=\frac{1}{t_{w}}
$$

where $t_{w}$ is the non-dimensional temperature evaluated at the wall. In accordance with [14] we compute the mean Nusselt number as the reciprocal of the weighted mean wall temperature.

\section{Results and Discussion}

\section{1. $H_{1}$ Case}

Fig. 2 presents the temperature profiles along a section through a square duct $(a / b=1)$ at $y=0$, for a range of Hartmann numbers. There is clearly the expected drop in temperature difference with increasing Hartmann number as the flow velocity is increasingly suppressed. The Nusselt numbers are computed for this case of uniform axial heat flux and uniform peripheral temperature for a range of Hartmann numbers. Unlike the non-MHD case, the profile of the flow field is not the same in both $x$ and $y$ directions. Indeed, the profiles perpendicular to the Hartmann and side walls have significantly different profiles, shown in [1]. One can view this in terms of individual wall mean Nusselt numbers. In Fig. 3 we see the mean Nusselt numbers for a Hartmann wall $\left(N u_{i}\right)$ and a side wall $\left(N u_{j}\right)$. As expected, for low Hartmann numbers, the results converge to the non-MHD case with a value of 3.608 [13, 14]. Both Nusselt numbers increase significantly as the electromagnetic forces begin to dominate the viscous forces $(\mathrm{Ha}>1)$. The Nusselt number for the Hartmann wall increases more rapidly (than that for the side layer) due to the thinner boundary layer which is $O\left(\mathrm{Ha}^{-1}\right)$ for the Hartmann wall and $O\left(\mathrm{Ha}^{-\frac{1}{2}}\right)$ for the side wall. For large $\mathrm{Ha}$, the velocity profile in Shercliff flow exhibits a flat core region, the velocity of which is asymptotically $O\left(\mathrm{Ha}^{-1}\right)$, as is shown in [1]. The wall shear stress is approximately the ratio of the core velocity to the boundary layer thickness, and as such is asymptotically $O(1)$ for the Hartmann wall. As a result, $N u_{i}$ reaches a plateau for large $\mathrm{Ha}$ and the heat transfer rate saturates, in general agreement with Blum et al. [4] for the case of a circular duct.

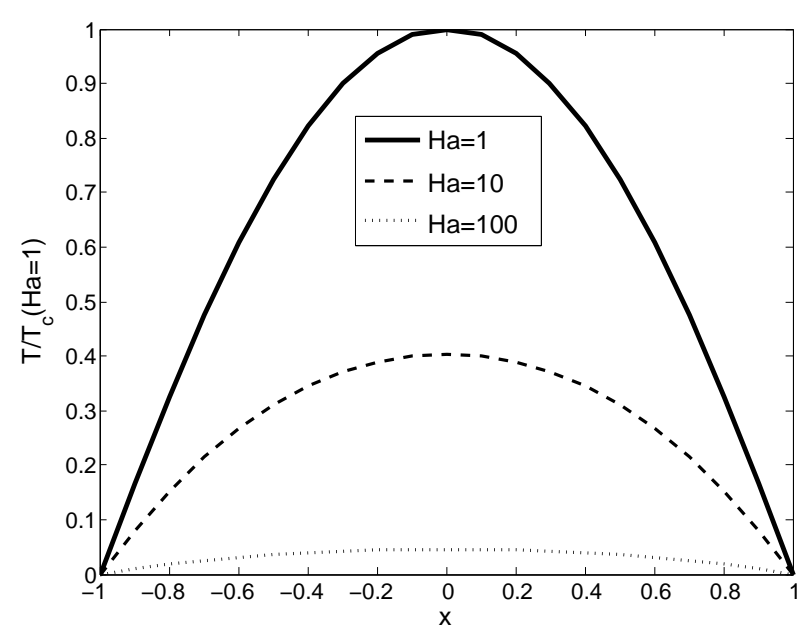

Figure 2: Temperature profiles across the midsection of a square duct

We now consider the effect of duct aspect ratio in the case of $b>a$. In Fig. 4 we present the overall mean Nusselt number (ie. the wall length-weighted sum of the wall Nusselt numbers) vs Hartmann number for a range of aspect ratios. In all cases the results reduce to the non-MHD case for $\mathrm{Ha}=0$, as shown in Table 1, where good agreement is obtained with other solutions. As expected, beyond $H a=1$, the electromagnetic forces dominate the viscous forces. As $b / a$ increases, the heat transfer is increasingly dominated by the relatively long Hartmann wall. The larger the aspect ratio, the more the heat transfer from the Hartmann wall dominates the heat transfer from the side wall and the steeper the rise in $N u$. For large $b / a$, a clear plateau is reached as the heat transfer rate at the Hartmann wall saturates. Alternatively, we could consider aspect ratios where $a>b$. 


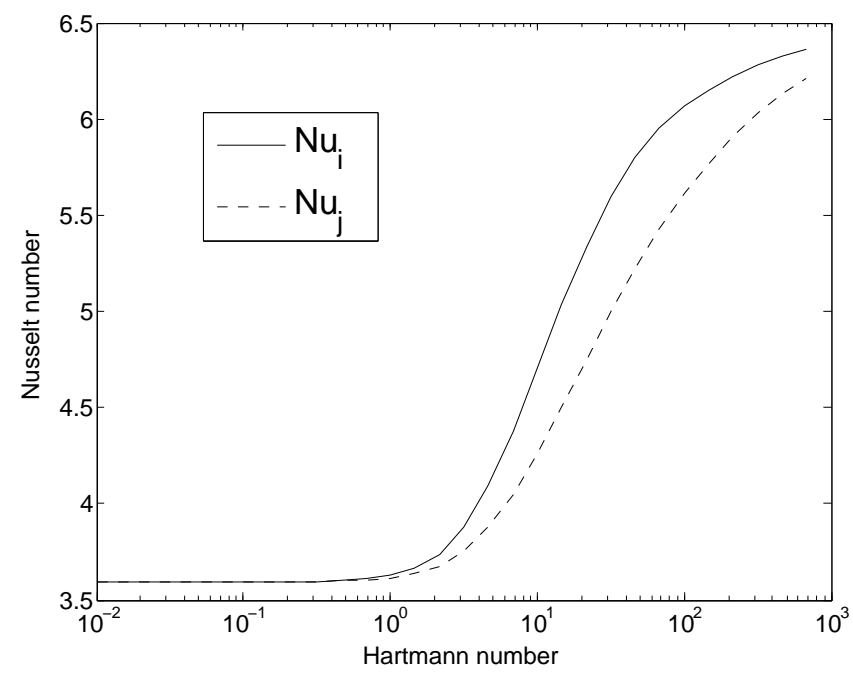

Figure 3: Mean Nusselt number vs Hartmann number

These results are shown in Fig. 5. In this case, as $a / b$ increases, the length of the Hartmann wall reduces relative to the side wall. The contribution to heat transfer by the Hartmann walls reduces with increasing aspect ratio, becoming dominated by the heat transfer through the side walls. For small $\mathrm{Ha}$, the shear stress at the side wall is essentially entirely due to viscous forces. As $H a$ increases, a Shercliff layer develops at the side wall, of thickness $O\left(\mathrm{Ha}^{-\frac{1}{2}}\right)$. Once this layer becomes thinner than the viscous layer, the shear stresses increase and increase the heat transfer. As the aspect ratio decreases further (for a fixed $d_{h}$ ), the shear stresses at the side wall due to the viscous forces must increase. These stresses are then only exceeded for a correspondingly thinner Shercliff layer and, inter alia, a larger $\mathrm{Ha}$. This is seen clearly from Fig. 5, where the onset of the MHD effect is progressively delayed for increasing aspect ratio. The slope of the curves in Fig. 5 are worthy of note: The Hartmann wall, with its higher wall shear stresses, provides better heat transfer per unit length than the side wall. The contribution of the Hartmann wall to overall heat transfer is dominant in the case of a square duct, resulting in a steep increase in $\mathrm{Nu}$ as $\mathrm{Ha}$ increases. As the aspect ratio increases, the Hartmann wall contributes less to the overall heat transfer and $\mathrm{Nu}$ increases as a function of $\mathrm{Ha}$ at a progressively slower rate, dictated by heat transfer through the side wall (see Fig. 3). This accounts, for instance, for the intersection of the cases $a / b=1$ and $a / b=2$.

Table 1: Comparison of $\mathrm{Nu}$ from literature against this paper for $H_{1}$ case for $\underline{\mathrm{Ha}=0}$

\begin{tabular}{cccc}
\hline Aspect Ratio & Shah and London [15] & Wang [16] & This Paper \\
\hline 1 & 3.608 & 3.6079 & 3.6068 \\
2 & 4.1233 & 4.1233 & 4.1220 \\
4 & 5.3311 & 5.331 & 5.3289 \\
6 & 6.0495 & 6.0494 & 6.0464 \\
8 & 6.4903 & 6.4903 & 6.4864 \\
\hline
\end{tabular}

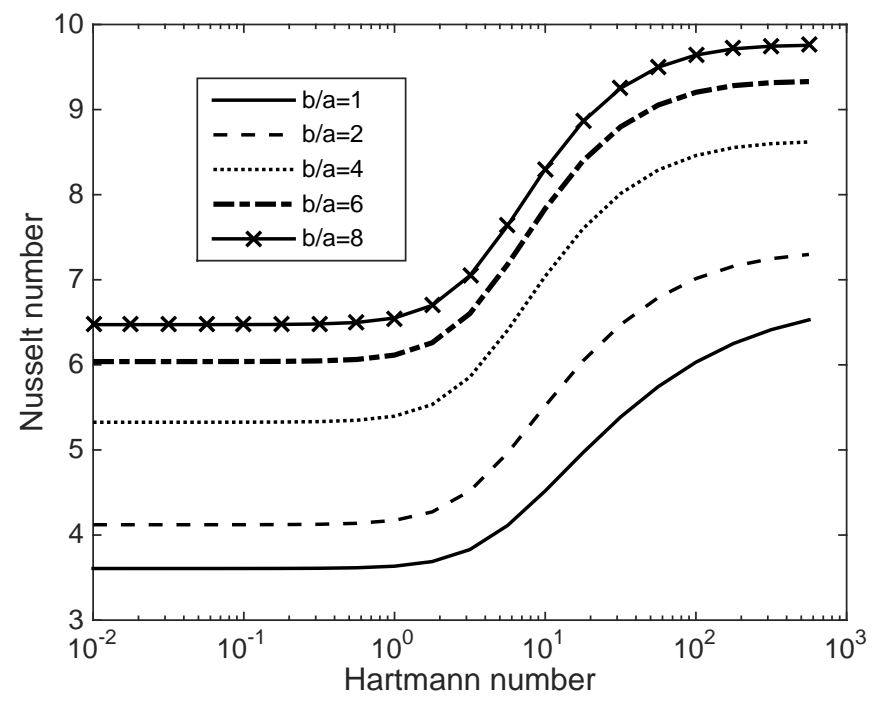

Figure 4: Overall Nusselt number vs Hartmann number for various aspect ratios $(b>a)$ for the $H_{1}$ case

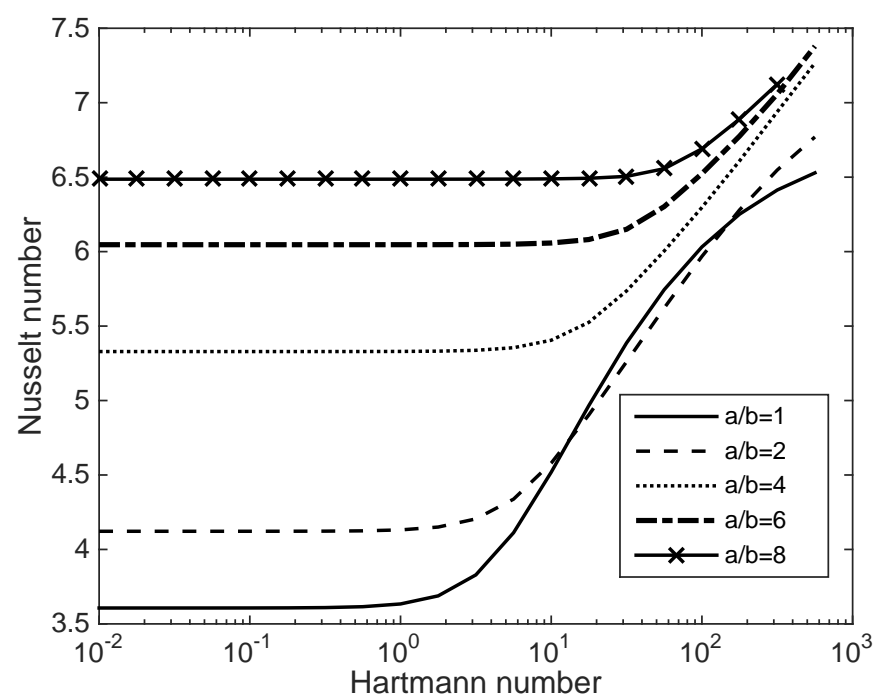

Figure 5: Overall Nusselt number vs Hartmann number for various aspect ratios $(a>b)$ for the $H_{1}$ case 


\section{2. $\mathrm{H}_{2}$ Case}

The expression for the $\mathrm{H}_{2}$ case involves numerous ratios of hyperbolic functions. For small aspect ratios and small Hartmann numbers, this expansion is used in its standard form and results are computed in MATLAB. For higher aspect ratios and Hartmann numbers, the arguments of these hyperbolic functions become large. This results in numerical errors and ultimately computational failure as the numbers involved exceed the normal machine precision. For such cases it is important to group these ratios and evaluate them by taking logarithms.

We first consider the effect of duct aspect ratio in the case of $b>a$. In Fig. 6 we present the overall mean Nusselt number vs Hartmann number for a range of aspect ratios. Note that the effect of aspect ratio is significantly reduced in the $\mathrm{H}_{2}$ case, relative to the $H_{1}$ case, with $N u \approx 3$. In all cases the results reduce to the non-MHD case for $H a=0$, as shown in Table 2, where again good agreement is obtained with other solutions. The heat transfer is largely dominated by the Hartmann wall and the Nusselt number increases with $\mathrm{Ha}$. The larger the aspect ratio, the more the heat transfer from the Hartmann wall dominates the heat transfer from the side wall and the steeper the rise in $N u$. Again, for large $b / a$, a clear plateau is reached as the heat transfer rate at the Hartmann wall saturates. For small $\mathrm{Ha}$, the Nusselt number decreases as the aspect ratio increases. However the heat transfer rate through the Hartmann wall increases more rapidly for high aspect ratios, as evidenced by the steeper gradient between $H a \approx 10$ and $H a \approx 100$.

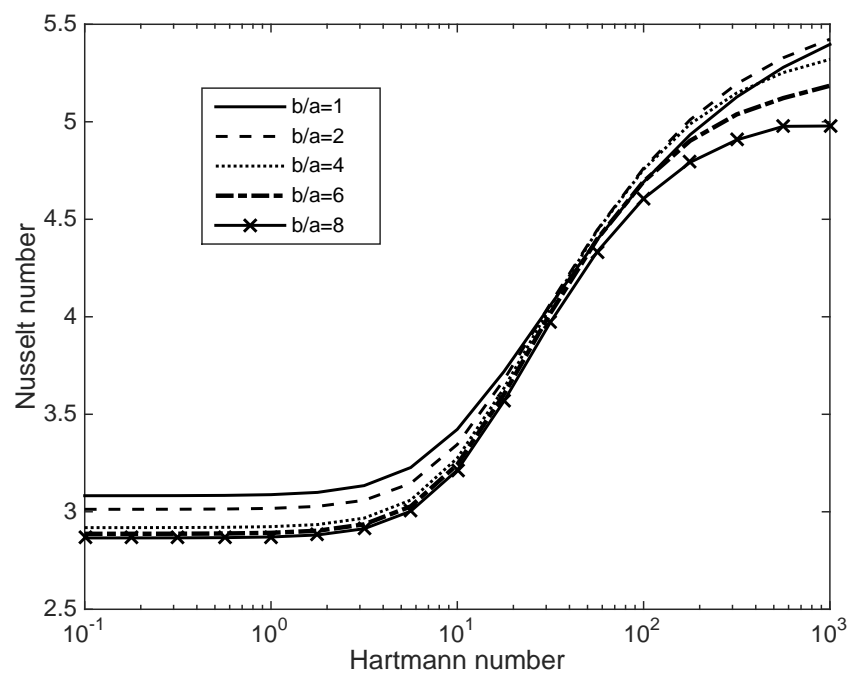

Figure 6: Overall Nusselt number vs Hartmann number for various aspect ratios $(b>a)$ for the $\mathrm{H}_{2}$ case

Alternatively, we consider the aspect ratio where $a>b$. These results are shown in Fig. 7. In this case, as the aspect ratio increases, the length of the Hartmann wall reduces relative to the side wall. Note also that the contribution to heat transfer of the Hartmann wall reduces with increasing aspect ratio in this $\mathrm{H}_{2}$ case. The general increase in Nusselt number with $\mathrm{Ha}$ is clear. However, for increasing aspect ratio, a minimum develops. This is an interesting feature and deserves some explanation.

Table 2: Comparison of $\mathrm{Nu}$ from literature against this paper for $\mathrm{H}_{2}$ case for $\mathrm{Ha}=0$

\begin{tabular}{cccc}
\hline Aspect Ratio & Spiga and Morini [17] & Wang [16] & This Paper \\
\hline 1 & 3.091 & 3.0873 & 3.0871 \\
2 & 3.022 & 3.0192 & 3.0187 \\
4 & 2.935 & 2.9326 & 2.9315 \\
6 & - & 2.9126 & 2.9102 \\
8 & 2.909 & 2.9074 & 2.9031 \\
\hline
\end{tabular}

In Fig. 8 we show the velocity profile for a modest Hartmann number $(H a=1)$, where MHD effects are negligible. The profile is relatively uniform over much of the $x$-direction. At $\mathrm{Ha}=25$, shown in Fig. 9, which corresponds to the minimum Nusselt number, Hartmann layers are developing along the shortest sides, but crucially the velocity profile in the $x$ direction becomes non-uniform and in particular velocity is suppressed near the short sides. The reasons for this are clear from Fig. 11 which shows the magnetic field in this orientation. The contour lines also represent the current paths and it is clear that for low to modest $\mathrm{Ha}$, that there is little or no current near the centre and concomitantly low velocity suppression. This is in marked contrast to the vertical orientation $(b>a)$ shown in Fig. 12. The effect of this is a suppression of the shear stress along the Shercliff walls near these shorter sides. Since the Hartmann walls are short with this aspect ratio, the heat transfer is dominated by the Shercliff layers and the high shear stresses in the Hartmann layers contribute little to the overall heat transfer. It is not until the Hartmann number increases sufficiently to produce a narrow Shercliff layer along the long side, that uniformity in the core region is re-established (shown in Fig. 10) and the subsequent high shear stress in the Shercliff layer, gives rise to increased heat transfer for $H a=1000$.

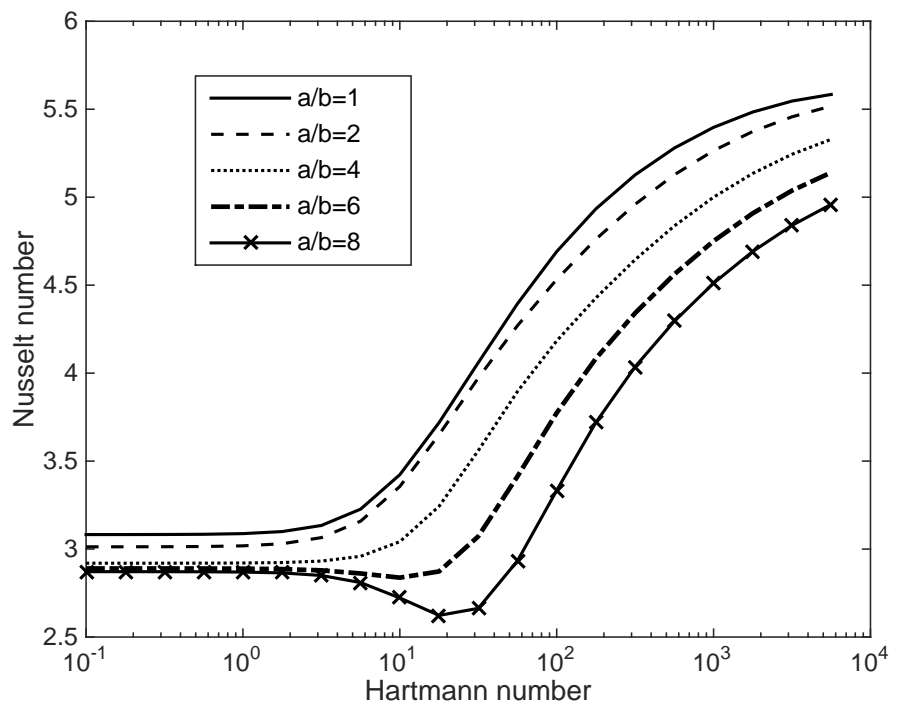

Figure 7: Overall Nusselt number vs Hartmann number for various aspect ratios $(a>b)$ for the $\mathrm{H}_{2}$ case 


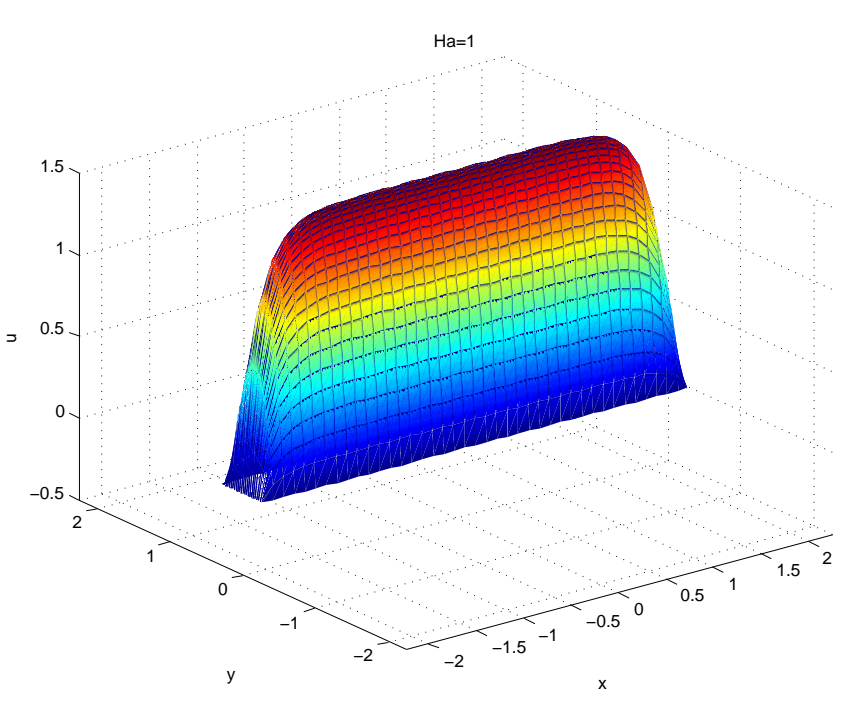

Figure 8: Velocity profile for $\mathrm{Ha}=1, \mathrm{a} / \mathrm{b}=8$

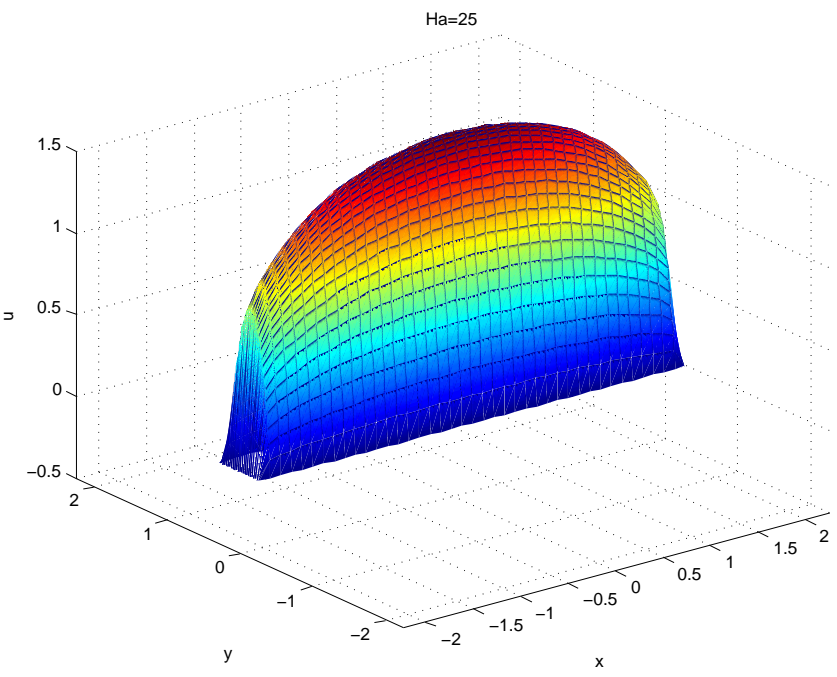

Figure 9: Velocity profile for $\mathrm{Ha}=25, \mathrm{a} / \mathrm{b}=8$ $\mathrm{Ha}=1000$

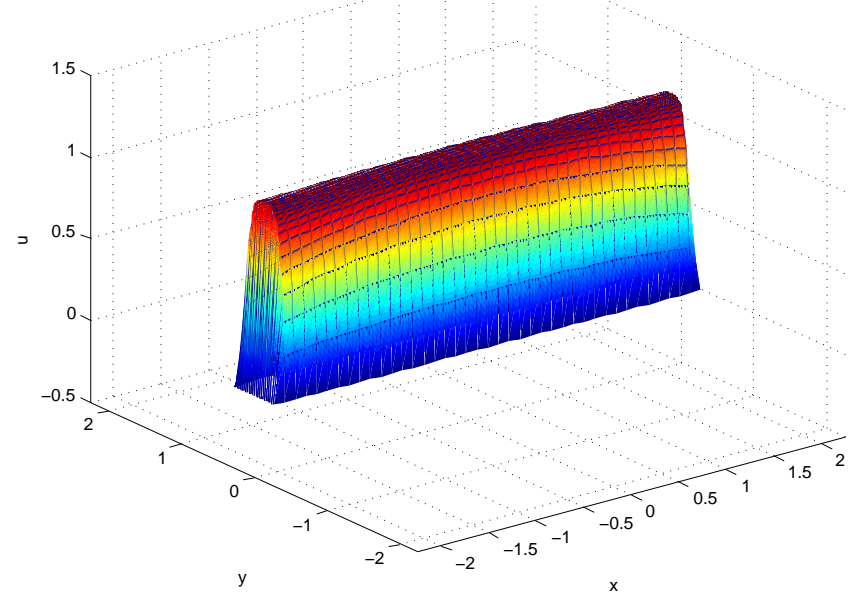

Figure 10: Velocity profile for $\mathrm{Ha}=1000, \mathrm{a} / \mathrm{b}=8$
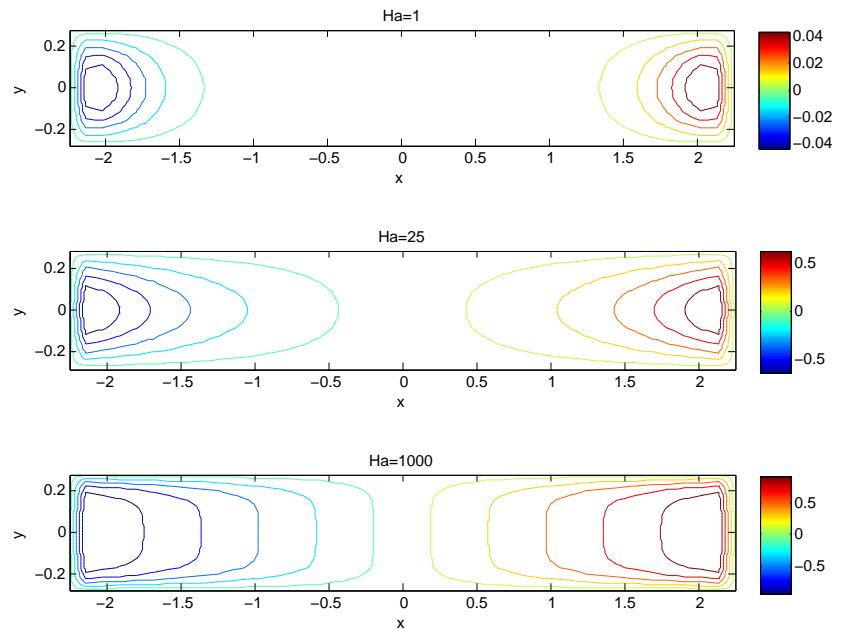

Figure 11: Induced magnetic fields, $a / b=8$

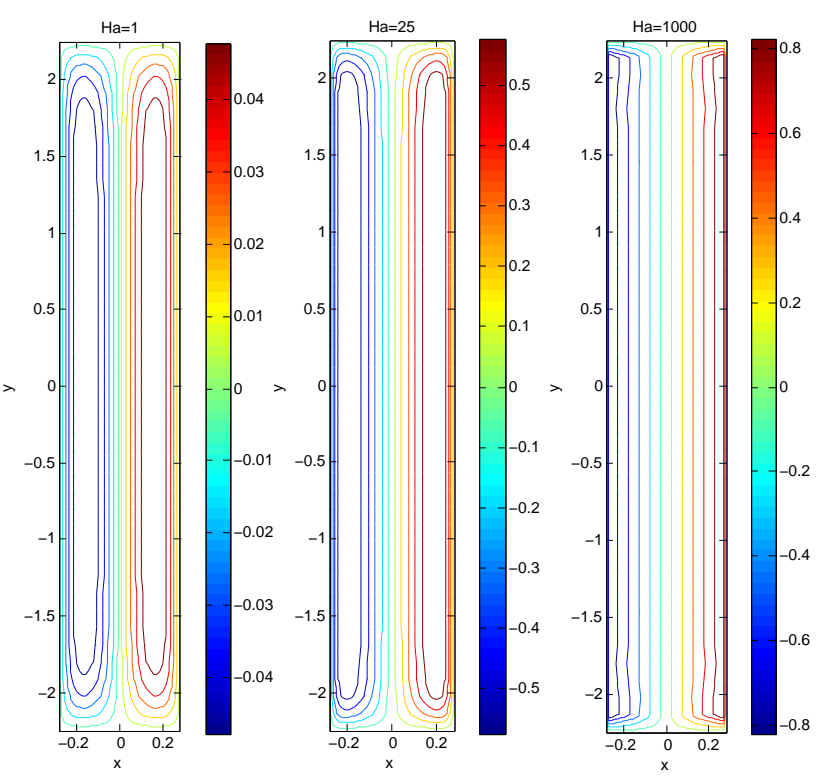

Figure 12: Induced magnetic fields, $\mathrm{b} / \mathrm{a}=8$

\section{Conclusions}

The heat transfer problem for MHD flow in rectangular ducts with electrically insulated walls subject to a transverse magnetic field is solved analytically for both $H_{1}$ and $H_{2}$ heat transfer cases. To the authors knowledge these results are new. Nusselt numbers are computed and show the expected results - notably convergence to the well known non-MHD values at low Hartmann number and saturation for high Hartmann number. Interestingly, in the $\mathrm{H}_{2}$ case for large aspect ratios where $a>b$, the Nusselt number has a minimum as a result of the distorted velocity distribution.

These results should prove useful for the validation of numerical codes where heat transfer effects are important - particularly in the design of liquid metal coolant fusion blankets. 


\section{Acknowledgements}

This work is supported by the Culham Centre for Fusion Energy (CCFE), Oxford, UK and Amec Foster Wheeler, UK.

\section{Appendix A. The Shercliff Solution}

The following Shercliff solution is valid for a rectangular duct $-a \leq x \leq a$ and $-b \leq y \leq b$ non-conducting walls.

$$
\begin{aligned}
& u(x, y)=\frac{H g}{R e} \sum_{n=1}^{\infty} u_{n}(x) \cos \lambda_{n} y \\
& h(x, y)=\frac{H g}{R e} \sum_{n=1}^{\infty} h_{n}(x) \cos \lambda_{n} y \\
& u_{n}(x)=\frac{k_{n}}{\lambda_{n}^{2} b}\left(1-\frac{\sinh p_{n_{2}} a \cosh p_{n_{1}} x-\sinh p_{n_{1}} a \cosh p_{n_{2}} x}{\sinh \left(p_{n_{2}}-p_{n_{1}}\right) a}\right) \\
& h_{n}(x)=\frac{k_{n}}{\lambda_{n}^{2} b}\left(\frac{\sinh p_{n_{1}} a \sinh p_{n_{2}} x-\sinh p_{n_{2}} a \sinh p_{n_{1}} x}{\sinh \left(p_{n_{2}}-p_{n_{1}}\right) a}\right) \\
& p_{n_{1}}=\lambda-\sqrt{\lambda^{2}+\lambda_{n}^{2}} \\
& p_{n_{2}}=\lambda+\sqrt{\lambda^{2}+\lambda_{n}^{2}} \\
& \lambda_{n}=\left(n-\frac{1}{2}\right) \frac{\pi}{b} \\
& \lambda=\frac{H a}{2} \\
& k_{n}=2 \frac{\sin \lambda_{n} b}{\lambda_{n}}
\end{aligned}
$$

\section{References}

[1] Shercliff, J., 1953. "Steady motion of conducting fluids in pipes under transverse magnetic fields". Mathematical Proceedings of the Cambridge Philosophical Society, 49(01), pp. 136-144.

[2] Hunt, J., 1965. "Magnetohydrodynamic flow in rectangular ducts". Journal of Fluid Mechanics, 21(04), pp. 577-590.

[3] Hunt, J., and Stewartson, K., 1965. "Magnetohydrodynamic flow in rectangular ducts. II". Journal of fluid mechanics, 23(03), pp. 563-581.

[4] Blūms, E., Mikhaŭlov, Y., and Ozols, R., 1987. Heat and Mass Transfer in MHD Flows. World Scientific Publishing.

[5] Zniber, K., Oubarra, A., and Lahjomri, J., 2005. "Analytical solution to the problem of heat transfer in an MHD flow inside a channel with prescribed sinusoidal wall heat flux". Energy Conversion and Management, 46(7-8), pp. 1147 - 1163.

[6] Ying, A., Lavine, A., and Tillack, M., 1989. "The effect of Hartmann and side layers on heat transfer in magnetohydrodynamic flow". Fusion Technol., 15.

[7] Sidorenkov, S., Hua, T., and Araseki, H., 1995. "Magnetohydrodynamics and heat transfer benchmark problems for liquid-metal flow in rectangular ducts". Fusion Eng. Des., 27, pp. $711-18$.

[8] Takase, K., and Hasan, M., 1995. "Heat transfer characteristics of rectangular coolant channels with various aspect ratios in the plasma-facing components under fully developed MHD laminar flow". 16th IEEE/NPSS Symposium Fusion Engineering, SOFE '95. Seeking a New Energy Era (Cat. No.95CH35852), vol.2, pp. $1538-41$.

[9] Tezer-Sezgin, M., 1994. "Boundary element method solution of MHD flow in a rectangular duct". Int. J. Numer. Methods Fluids, 18(10), pp. 937 -52 .
[10] Al-Khawaja, M. J., and Selmi, M., 2009. "Numerical solutions of two heat transfer limits of MFM square duct flow using matlab program". International Journal for Computational Methods in Engineering Science and Mechanics, 10(1), pp. 102-107.

[11] Al-Khawaja, M., and Selmi, M., 2006. "Highly accurate solutions of a laminar square duct flow in a transverse magnetic field with heat transfer using spectral method". Journal of Heat Transfer, 128(4), pp. 413-417.

[12] Lahjomri, J., Zniber, K., Oubarra, A., and Alemany, A., 2003. "Heat transfer by laminar Hartmann's flow in thermal entrance region with uniform wall heat flux: the Graetz problem extended". Energy Conversion and Management, 44(1), pp. 11-34.

[13] Shahmardan, M. M., Norouzi, M., Kayhani, M. H., and Amiri Delouei, A., 2012. "An exact analytical solution for convective heat transfer in rectangular ducts". Journal of Zhejiang University SCIENCE A, 13(10), pp. 768-781.

[14] Morini, G. L., 2000. “Analytical determination of the temperature distribution and Nusselt numbers in rectangular ducts with constant axial heat flux". International Journal of Heat and Mass Transfer, 43(5), pp. 741755.

[15] Shah, R., and London, A., 1978. Advances in Heat Transfer: Supplement 1, Laminar Forced Flow in Ducts. Advances in Heat Transfer. Academic Press, New York.

[16] Wang, C., 2014. "On the nusselt number for $\mathrm{H} 2$ heat transfer in rectangular ducts of large aspect ratios". Journal of Heat Transfer, 136(7), p. 074501.

[17] Spiga, M., and Morini, G., 1996. "Nusselt numbers in laminar flow for H2 boundary conditions". International Journal of Heat and Mass transfer, 39(6), pp. 1165-1174. 